\title{
A global analysis of CNVs in diverse yak populations using whole-genome resequencing
}

\author{
Hui Wang ${ }^{1 \dagger}$, Zhixin Chai ${ }^{1 \dagger}$, Dan Hu' , Qiumei $\mathrm{Ji}^{2}$, Jinwei Xin², Chengfu Zhang ${ }^{2}$ and Jincheng Zhong ${ }^{1 *}$ (D)
}

\begin{abstract}
Background: Genomic structural variation represents a source for genetic and phenotypic variation, which may be subject to selection during the environmental adaptation and population differentiation. Here, we described a genome-wide analysis of copy number variations (CNVs) in 16 populations of yak based on genome resequencing data and CNV-based cluster analyses of these populations.

Results: In total, we identified 51,461 CNV events and defined 3174 copy number variation regions (CNVRs) that covered $163.8 \mathrm{Mb}(6.2 \%)$ of yak genome with more "loss" events than both "gain" and "both" events, and we confirmed 31 CNVRs in 36 selected yaks using quantitative PCR. Of the total $163.8 \mathrm{Mb}$ CNVR coverage, a 10.8 Mb region of highconfidence CNVRs directly overlapped with the $52.9 \mathrm{Mb}$ of segmental duplications, and we confirmed their uneven distributions across chromosomes. Furthermore, functional annotation indicated that the CNVR-harbored genes have a considerable variety of molecular functions, including immune response, glucose metabolism, and sensory perception. Notably, some of the identified CNVR-harbored genes associated with adaptation to hypoxia (e.g., DCC, MRPS28, GSTCD, MOGAT2, DEXI, CIITA, and SMYD1). Additionally, cluster analysis, based on either individuals or populations, showed that the CNV clustering was divided into two origins, indicating that some yak CNVs are likely to arisen independently in different populations and contribute to population difference.
\end{abstract}

Conclusions: Collectively, the results of the present study advanced our understanding of CNV as an important type of genomic structural variation in yak, and provide a useful genomic resource to facilitate further research on yak evolution and breeding.

Keywords: Bos grunniens, CNV, Environmental adaptation, Cluster analysis

\section{Background}

Copy number variation (CNV), defined as deletion or duplication of DNA fragments larger than $50 \mathrm{bp}$ in length compared with a reference genome, is a ubiquitous form of genomic structural variation $[1,2]$, and has pronounced effects on phenotype $[3,4]$ and evolutionary adaptation [5, 6] through altering gene expression levels or transcript structure. Many previous publications have reported the effects of CNVs on evolution [7], population diversity $[8,9]$, and various physiological

\footnotetext{
* Correspondence: zhongjincheng518@126.com

${ }^{\dagger}$ Hui Wang and Zhixin Chai contributed equally to this work.

'Key Laboratory of Qinghai-Tibetan Plateau Animal Genetic Resource

Reservation and Utilization (Southwest Minzu University), Ministry of

Education, Chengdu 610000, People's Republic of China

Full list of author information is available at the end of the article
}

processes(e.g., lipid metabolism $[4,10]$ and glucose metabolism [11]) and pathological processes (e.g., cancer related biologic processes [12] and the occurrence and progression of many tumors [13]). Furthermore, CNV provides the mechanisms and resources for creating new genes [14].

Recent studies on the distribution of CNVs in the human genome have shown that more than $12 \%$ of the human genome containing CNVs [15]. Given the ubiquitous distribution of CNVs and their importance, advances in $\mathrm{CNV}$ detection have extended to livestock and poultry species, including pigs [16], goats [17], sheep [18], cattle [19], and chicken [20]. These animal datasets provide a very valuable resource for evolution and genetic improvement research. Interestingly, there is growing evidence for $\mathrm{CNVs}$ associated with production traits

(c) The Author(s). 2019 Open Access This article is distributed under the terms of the Creative Commons Attribution 4.0 International License (http://creativecommons.org/licenses/by/4.0/), which permits unrestricted use, distribution, and 
and environmental adaptation. For example, in Holsteins cattle, $34 \mathrm{CNVs}$ on 22 chromosomes have been characterized as being significantly associated with milk production traits, some of which are located within or near known QTL for milk production traits [21]. CNVs of the relaxin/insulin-like family peptide receptor 4 gene and the olfactory receptor are strongly associated with residual feed intake in Holstein cattle [22], whereas the $\mathrm{CNV}$ region of glycerol-3-phosphate acyltransferase 2 gene shows associations with carcass length and fatty acid composition in backfat and intramuscular fat [23]. Furthermore, the agouti signaling protein gene $\mathrm{CNV}$ allele has been shown to be almost entirely associated with coat color in Tibetan sheep [24]. $9805 \mathrm{CNV}$ regions (CNVRs) are estimated to cover approximately 13.05\% of the cattle genome and overlap with 5495 genes that are involved in environmental adaptation of Nellore cattle to tropical areas [25]. Apolipophorin 3 and fatty acid-binding protein 2 , two genes involved in lipid transport and metabolism, are highly duplicated in the beef breeds [26]. These findings indicate that multiple beneficial CNVs may have been naturally selected in livestock during adaptation to different environments and could be associated with population diversity and economic characteristics.

The molecular mechanism underlying hypoxic adaptation in highland-local-animal has aroused attention for biological and medical research, not only because of the evolutionary significance of high-altitude adaptation, but also to understand human hypoxia-related diseases (e.g., acute mountain sickness, high-altitude pulmonary edema, and high-altitude cerebral edema [27]). For animal migrating to or living in high-altitude regions, environmental hypoxia is a primary challenge. The yak (Bos grunniens), a ruminant that separated from other ruminants approximately 2.2 million years ago, is mainly distributed in Qinghai-Tibetan Plateau (QTP) at altitudes ranging from 2500 to $6000 \mathrm{~m}$, a region characterized by no absolutely frost-free period. It is known that yak is the only major livestock animal that can survive the extremely harsh environments of QTP, and are noted for their ability to tolerate the cold and anoxic conditions and resist the local disease threats. At present, there are more than 16 million domestic yaks, which provide the necessities for Tibetans and other nomadic pastoralists in high-altitude environments. In addition, there are 18 affirmed populations of the species in China, including the artificially breed Datong yak. It should be noted that the domestic yak is the only large animal that still coexists with its wild ancestors in similar environments [28]. Therefore, the yak provides a good framework for studying effects of $\mathrm{CNV}$ in hypoxic adaptation in large livestock. In previous studies, $161 \mathrm{CNV}$ regions were detected from two yak individuals using the cattle-specific Nimblegen3x720K comparative genomic hybridization (CGH) array, and on the basis of a comparison between domestic and wild yak populations, Zhang et al. identified 121 potentially selected CNV regions harboring genes related to neuronal development, reproduction, nutrition and energy metabolism [29].

To date, few studies have confirmed the genome-wide presence of CNVs in relation to hypoxic adaptation in yak. Here, we selected 16 yak populations from different altitudes to conduct a genome-wide CNV analysis, and subsequently performed cluster analysis at both individual and population level. Initially, we conducted genome-wide CNV screening of the 16 yak populations, and thereafter performed GO enrichment analysis of CNVR-harbored genes to identify their functions. The information gained in this study will constitute a valuable resource for different yak populations for future studies on phenotypic variation and breeding, and will also provide important insights into the mechanisms underlying yak genomic evolution.

\section{Methods}

\section{Tissue samples}

Forty-eight healthy four-year-old yaks of similar weight were selected from 16 populations inhabiting widely distributed locations across the QTP (Additional file 1). The 16 studied yak populations were as follows: Dingqing (DQ), Shenzha (SZ), Gongbujiangda (GD), Cuona (CN), Jinchuan (JC), Zhongdian (ZD), Sibu (SB), Leiwuqi (LWQ), Pali (PL), Maiwa (MW), Gannan (GN), Jiulong (JL), Tianzhu (TZ), Datong (DT), Bazhou (BZ), Jiali (JL1). It should be noted that the Datong yak is an artificial breed, the founding male parent of which was a wild yak. For each population, triangular skin biopsy samples (ear notches) measuring approximately $15 \mathrm{~mm}$ were obtained from three individuals by using a pig ear notcher, Hemostasis at the biopsy site was accomplished by applying a spring paper clip over cotton gauze for $5 \mathrm{~min}$ [30]. The samples were immediately snap-frozen in liquid nitrogen.

\section{Construction of sequencing library, sequencing, and data processing}

Genomic DNA was extracted and purified following the standard phenol/chloroform extraction procedure [31], and subsequently quantified using an Aliglent 2100 bioanalyzer (Agilent Technologies, Palo Alto, CA) and agarose gel electrophoresis [29, 32]. Paired-end sequencing libraries with an insert size of $500 \mathrm{bp}$ were sequenced using an Illumian Hiseq 2000 platform.

Low-quality reads were filtered out using PRINSEQ (version 0.20.4) to obtain the clean reads in accordance with previously reported criteria [33]. Briefly, the 
following reads were removed: (1) reads with $\geqq 10 \%$ unidentified nucleotides; (2) reads for which more than $60 \%$ of the read length had a Phred quality value $\leqq 7$; (3) reads with more than $10 \mathrm{bp}$ aligned to the adapter; (4) duplicate reads; (5) reads with three consecutive base pairs for which Phred value was lower than 14; (6) reads shorter than $45 \mathrm{bp}$. The clean reads were mapped to the yak reference genome (BosGru_v2.0) using BWA-MEM (0.7.15-r1140) with set default parameters, and then the SAM format results were sorted and indexed into Binary Alignment Map (BAM) format files using SAM tools. Finally, Genome Analysis Toolkit (version 4.0.10.1) was used to realignreads located in regions around indels to reduce the inaccurate alignment.

\section{CNV identification and CNVR determination}

The software CNVnator (v0.3.2), which is better adapted than other similar softwares with respect to the accuracy of the copy number estimation [34], was used for realigned BAM file processing. The parameters were selected following the recommendations of the author. After setting the sliding window to a fixed value of 100 $\mathrm{bp}$, the following steps were run to calculate the number of reads in the interior and both sides of the window: CNVnator-tree, CNVnator-his, CNVnator-stat, CNVnator-partition, and CNVnator-cal. Thereafter, comparison of the average depth of re-sequencing data and the reference genome was used for further correction, thereby identifying the occurrence of $\mathrm{CNV}$ in the preset window.

To avoid bias caused by different coverage, the existence of undefined nucleotide $(\mathrm{N})$ in the genome sequence, and false positives results of the CNVnator software, the CNVs obtained from previous steps were used for subsequent analysis. The average coverage depth for each sample and $\mathrm{CNV}$, and the ratio of non- $\mathrm{N}$ bases for each $\mathrm{CNV}$ were calculated. For the deletion type of CNVs, the CNVs were selected as clean CNVs, which should satisfy the following conditions: (1) a ratio of non- $\mathrm{N}$ bases greater than 0.4 and the covered bases more than $50 \%$ of non- $\mathrm{N}$ bases; (2) an average coverage depth less than $60 \%$ of that in the sample. Condition for selection of the duplication type of CNVs were as follows: (1) a ratio of non- $\mathrm{N}$ bases greater than 0.9 , with covered bases representing more than $90 \%$ of non-N bases; (2) an average coverage depth greater than 140\% of that in the sample. Those CNVs satisfying the respective two conditions were selected as clean CNVs. In addition, we only retained the CNVs longer than $1.5 \mathrm{~kb}$ for further analysis.

The CNV region is defined as a combined region of overlapping CNVs on the yak genome. CNVRs are merged from different individuals with any amount of overlap by extending the boundaries of the overlapping CNVs. Here, Perl script was used for defining the CNVRs. Only CNVRs present in more than three individuals were used for subsequent functional and comparative analysis.

\section{Quantitative PCR (qPCR) and resequencing data validation}

Quantitative PCR analysis was performed to validate the accuracy of the CNV assignments. 12 CNVRs that encompassed functional genes were selected for validation in 24 randomly-selected individuals. The bovine basic transcription factor 3 (BTF3) gene, for which no CNVs or segmental duplications (SDs) were detected in our analysis and in previous studies [29, 33], was selected as a reference location for $\mathrm{qPCR}$ validation. The $\mathrm{M}$-Value and V-value for BTF3 were 0.25 and 0.11 , respectively, and thus the gene is considered to be very stable, and the normalization factor is reliable according to the thresholds suggested by Vandesompele et al. (i.e., $\leq 1.5$ for $\mathrm{M}$-value and $\leq 0.15$ for $\mathrm{V}$-value) [35]. The primers used for qPCR amplification were designed using Primer Premier 5.0 (Premier, Canada) software and synthesized by Invitrogen (Shanghai, China). These primers are listed in Additional file 2.

Real time qPCR assays were performed using SYBR Premix Ex Taq II(Perfect Real Time, Takara, Japan) according to the manufactures' instructions. Gene expression was normalized to that of the reference BTF3 gene. All real-time reactions, including controls with no templates, were carried out using a Bio-Rad CFX96 real-time PCR detection system (Bio-Rad, USA). Relative expression was calculated using the $2^{-\Delta \Delta \mathrm{Ct}}$ method. Mean expression levels and standard deviations were obtained from three independent experiments.

\section{SDs detection and association with the distribution of CNV}

Using yak BosGru_v2.0 genome assembly, a whole-genome assembly comparison approach was applied to detect putative SDs. Briefly, sequence identified as SDs should fulfil the conditions that the sequence is larger than $1 \mathrm{~kb}$ in length and has identity greater than 90\%. The overlap between the SDs and CNVR was calculated using custom Perl script. Chi-square analysis of SD distribution in the genome and in CNVRs was then performed using the Chi.test package in $\mathrm{R}$ (version 3.3.1). In addition, using previously published Perl script [36], the association between CNVs and SDs was examined via random simulations.

\section{Gene annotation and ontology}

To assess the gene in each CNVR, the coordinates of each CNVR in the yak genome assembly were determined and gene annotation was performed. For this 
analysis, we used those genes comprising more than 50\% CNVR. Gene ontology (GO) enrichment analysis was performed using the online tool DAVID (https://david.n cifcrf.gov/). $P$ values were adjusted by false-discovery rate (FDR). GO terms associated with CNVRs and whole genome background were plotted using WEGO online software [37] (http://wego.genomics.org.cn/).

\section{Heatmap analysis}

Heatmap analysis was performed based on the sequencing depth obtained for each individual. Using the "depth" command in SAM TOOLS, the depth of each base was computed for each sample. The ratio of the average depth of each window to the effective depth for each individual was calculated as the estimated copy number. The estimated copy number values for all samples were then plotted using the heatmap function in the R package.

Cluster analysis between different populations based on CNV To identify CNV genes with high differentiation among the 16 populations surveyed in this study, the status of each $\mathrm{CNV}$ in each sample was defined as follows: deletion-type CNVs were designated -1 and the amplification-type $\mathrm{CNVs}$ were designated 1 . If no $\mathrm{CNV}$ were detected, this condition was designated 0 . Using these values, we constructed a CNV matrix for each sample for clustering. Subsequently, we integrated the values obtained for three individuals in each population, and reconstructed a matrix for cluster analysis among populations. The metric formula used in this analysis has been reported previously [33].

\section{Statistics}

The $\mathrm{R}$ package q-value (version 3.3.1) was used to calculate the FDR, and threshold for significant associations was set at a q-value of $<0.05$.

\section{Results}

\section{Genome-wide identification of CNVs}

A total of $0.98 \mathrm{Tbp}$ sequences with an average depth of $8.1 \times$ was obtained from the 48 individuals examined (Additional file 1). Aligning these sequences to the yak reference genome revealed that reads from single individual covered at least $67.5 \%$ of the genome and, on average, $75.69 \%$ of the reference genome was covered, indicating that the data are sufficient and of sufficiently high quality for CNV detection.

The SNP distribution of the 48 individuals was obtained via comparing the detected genotype to polymorphism sites in the reference sequence. In total, we detected 247,811,300 SNP events among the 48 individuals, with an average number of 5,162,735 SNPs per individual (Additional file 3). Using CNVnator software based on the RD method [34], we detected a total of $51,461 \mathrm{CNV}$ events (with an average of 24,729 gain and 26,732 loss events) from the 48 individuals (Fig. 1, Additional file 4), the average number of CNVs per individual was 1072 with an average of 557 gain and 515 loss events, and the average number of specific CNV events per individual was 107. The size of the CNVs identified varied from $1.5 \mathrm{~kb}$ to $1460.6 \mathrm{~kb}$, with an average size of $12.26 \mathrm{~kb}$. Details of the identified CNVs and location information for each individual are listed in

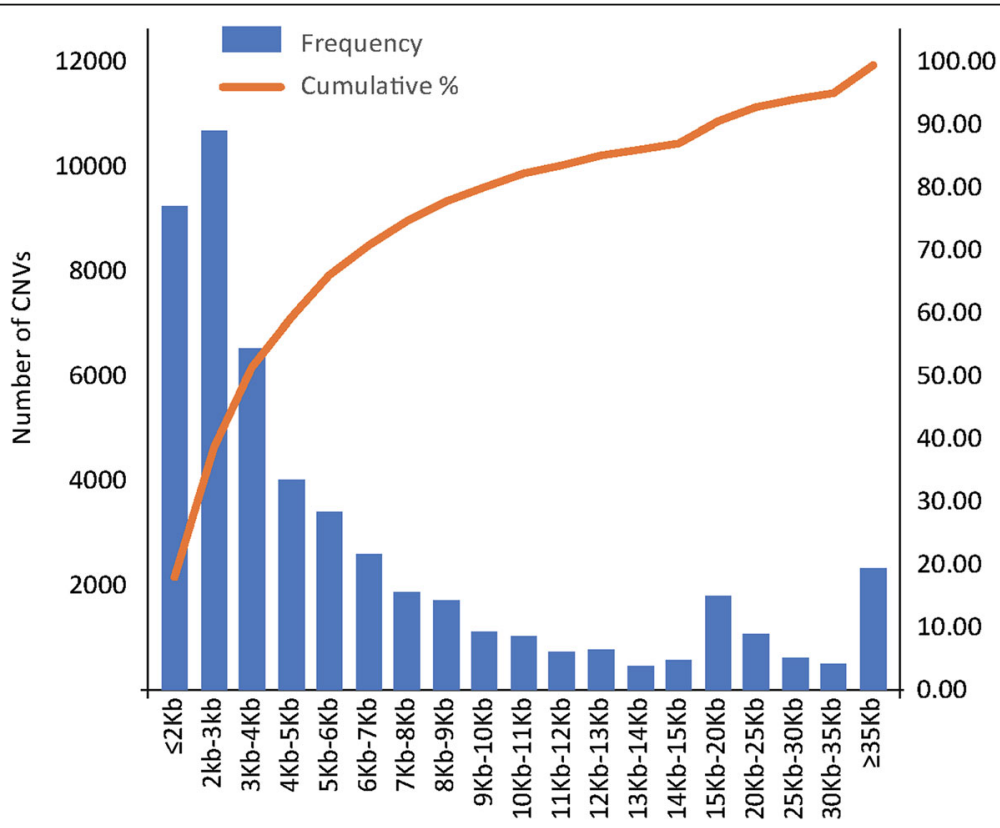

Fig. 1 CNV size interval distribution, the average CNV size is $12.26 \mathrm{~kb}$ 
Additional file 5. In addition, we found that CNVs were distributed in a non-random way and their contents vary across chromosomes.

A total of 3154 CNVRs were defined by merging all overlapping calls across multiple individuals into unique regions and filtering out those that were present in fewer than three individuals (Additional file 6). These CNVRs occupied $163.8 \mathrm{Mb}$ or $6.2 \%$ of the yak genome (Fig. 2). Furthermore, 28 CNVRs were found to be common to all 48 individuals. The detected CNVRs were divided into three categories according type: 1077 gain, 1776 loss, and 301 gain and loss (Additional file 6).

qPCR analysis was performed to evaluate the accuracy of individual CNVs predicted [38]. We accordingly found that $12 \mathrm{CNVRs}$ overlapped functional genes and 24 different individuals were randomly selected for validation. The results showed that $89 \%$ of the CNVs $(32 / 36)$ had an accurate copy number (Additional file 2).

\section{SDs detection and comparison with CNVRs}

On the basis of whole-genome assembly comparison (WGAC) methods [39], we initially identified 27,705 putative SD events (Table 1). In this regard, it is interesting to note that of the $163.8 \mathrm{Mb}$ CNVRs distributed across the yak genome, $10.8 \mathrm{Mb}$ of high-confidence CNVRs directly overlapped with $52.9 \mathrm{Mb}$ SDs.

\section{Functional analysis of CNV-harbored genes}

In total, 1374 protein-coding genes within or partially encompassed by the 3154 identified CNVRs were retrieved from the current yak genome (Additional file 7). In order to obtain insight into the biological functions of the CNVR-harbored genes, GO enrichment was performed using the DAVID bioinformatics resource. GO analysis annotated 765 of the 1374 genes in three main GO categories: molecular function, cellular component and biological process (Table 2). Genes in all categories were mainly involved in 23 processes, including transmembrane transporter activity [e.g., deleted in colorectal cancer $(D C C)$ ], guanyl ribonucleotide binding [mitochondrial ribosomal protein s28 (MRPS28)], purine metabolism, glucose metabolism [e.g., mono-acylglycerol O-acyltransferase 2 (MOGAT2)], immune response [e.g., dexi homolog (DEXI), class II major histocompatibility complex transactivator (CIITA), and SET and MYND domain containing 1(SMYD1)], sensory perception of chemical stimulus (e.g., dopamine receptor D3), and sensory perception (e.g., solute carrier family 26 member 4 gene). This set of CNV-harbored genes has a wide spectrum of molecular functions, cellular components and biological processes, and provides a rich resource for validating hypotheses on the genetic basis of phenotypic variation within and among different yak populations. Furthermore, it is worth noting that a series of
CNVR-harbored genes that are associated with adaptation to high altitude [e.g., DCC, glutathione S-transferase C-terminal domain containing (GSTCD), MRPS28, and MOGAT2] showed significant differences in copy number among different populations living at different altitudes (Fig. 3).

\section{Cluster analysis among individuals based on CNV}

Using 48 selected yaks, we determined the different distribution events of CNVs in which we designated a copy number deletion event as -1 , an amplification event as 1 , and a non-CNV as 0 , and subsequently performed cluster analyze among the different individuals (Additional file 6). The results showed that the $\mathrm{CNV}$ clustering was divided into two branches (Fig. 4). Furthermore, cluster analyses were performed based on 16 populations (Fig. 5), and the results further indicated that the 16 populations were consistently classified into two continental groups.

\section{Discussion}

In this study, we detected and verified CNVRs using whole-genome resequencing and $\mathrm{qPCR}$, the results showed that the CNVRs occupied $163.8 \mathrm{Mb}$ or $6.2 \%$ of the yak genome, which is slightly higher than the value determined in previous study that examined 14 wild and 65 domestic yaks (153 Mb, 5.7\%) via resequencing [33] and considerably higher that than determined for two yak individuals (33 Mb, 1.25\%) based on a CGH array approach [29]. Due to differences in the technology employed and the individual samples used for CNVs analysis, even though we found that the number trend of the three categories was: loss CNVRs > gain CNVRs > gain and loss CNVRs, it is difficult to compare the CNVs detected in different studies. In the present study, using next-generation sequencing, we achieved greater confidence and better resolution in calling CNVs than has been obtained previously. Therefore, compared with previous studies, most of these newly discovered CNVRs are novel, and thus further supplement the research base of CNVs in yak. In addition, for the first time, our study focuses on the genome CNV maps of different yak populations.

The validation of the accuracy of individual CNVs by qPCR showed that $89 \%$ of the CNVs had an accurate copy number. It should be note, however, that, given the complex structure of CNVRs and low-copy duplications with lower sequence similarities, false positive identification is common in CNV detection via $\mathrm{qPCR}$ analysis $[29,40]$. Furthermore, the quality of the assembled reference and the annotated repeats plays a key role in discovering CNVs using the RD method [26]. Consequently, in order to preclude false positives from analysis, fluorescence in situ hybridization and array comparative genomic hybridization $[41,42]$ will be required to obtain more accurate information in further studies. 


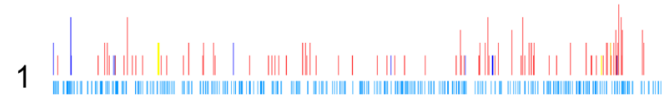

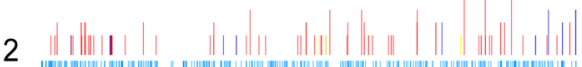

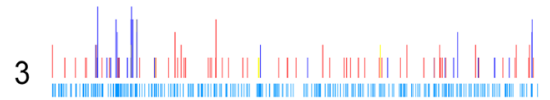

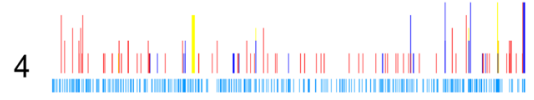

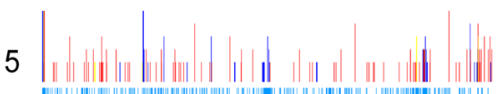

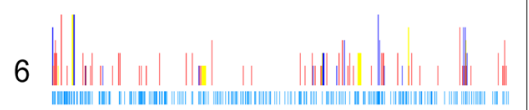

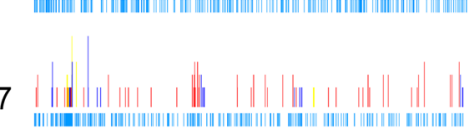

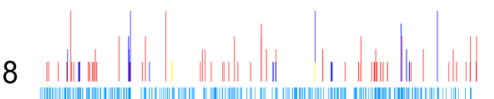

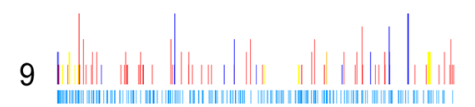

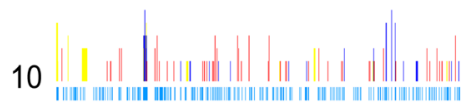

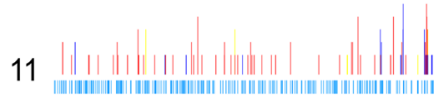

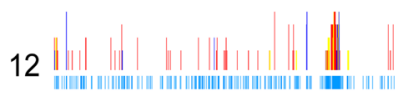

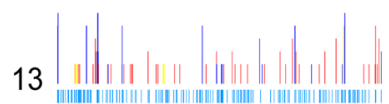

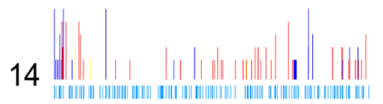

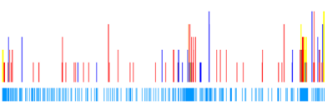

15

$\left|\|\|_{\|}\right|+||||||,\left|\||,|, \mid\|_{\|}\right.$

16

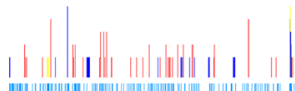

17

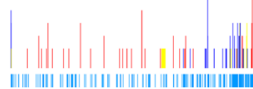

18

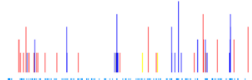

19

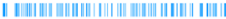
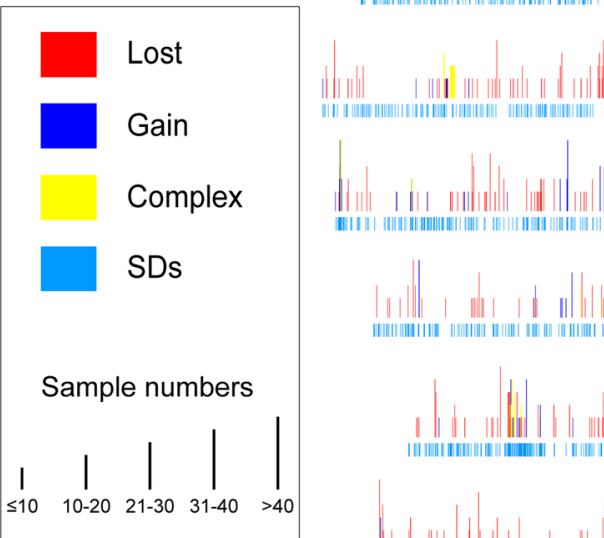

20

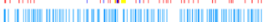

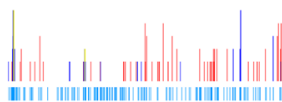

21

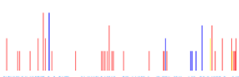

22

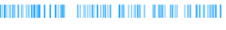

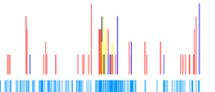

23

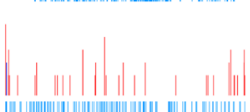

24

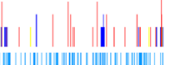

25

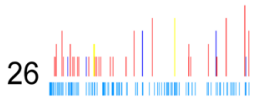

||||||||||$||||$,

27

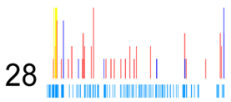

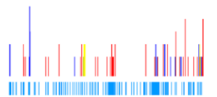

29

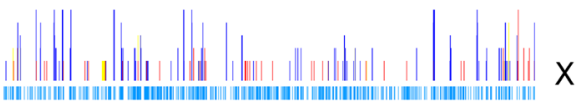

Fig. 2 Genomic landscape of CNVRs and SDs in yak. The SDs are plotted as light blue bars. The CNVRs are illustrated above the SDs in rad (loss), navy blue (gain) and yellow (complex of gains and losses). The chromosome numbers $(1-29, X)$ are shown nearby correspond CNV landscape, and the bar height represents different sample numbers $(\leq 10 、 10-20 、 21-30 、 31-40 、>40)$

Table 1 The summary of SDs in yak

\begin{tabular}{llll}
\hline Length (bp) & Events & Average length (bp) & Coverage (Mp) \\
\hline 1000 & 26,945 & 1615 & 45.199657 \\
5000 & 718 & 5990 & 6.827168 \\
10,000 & 42 & 11,097 & 0.875054 \\
\hline
\end{tabular}

Taking into consideration that segmental duplications (SDs) are among the major catalysts and hotspots for CNV formation in mammals [43], we sought to determine whether there are association between the CNVs and SDs in yak. After analysis, we found that $10.8 \mathrm{Mb}$ of the 163.8 $\mathrm{Mb}$ CNVRs directly overlapped with $52.9 \mathrm{Mb}$ SDs. We speculate that the SDs distribution pattern is predominant in yak CNVs, which is consistent with previous studies showing that CNVs are enriched with SDs [44].

Our finding that the CNV-harbored genes are enriched in sensory perception is consistent with the 
Table 2 The significant GO categories of CNVR-harbored genes

\begin{tabular}{|c|c|c|c|c|c|}
\hline GO ID & Function & GO type & Adjusted $p$-value & $\begin{array}{l}\text { Number of CNV } \\
\text { harbored genes }\end{array}$ & $\begin{array}{l}\text { Number of all } \\
\text { yak genes }\end{array}$ \\
\hline GO:0015399 & primary active transmembrane transporter activity & molecular function & 0.000469 & 11 & 50 \\
\hline GO:0019001 & guanyl nucleotide binding & molecular function & 0.017441 & 33 & 328 \\
\hline GO:0032561 & guanyl ribonucleotide binding & molecular function & 0.024169 & 31 & 312 \\
\hline GO:0016887 & ATPase activity & molecular function & 0.038654 & 20 & 191 \\
\hline GO:0004871 & signal transducer activity & molecular function & 0.087474 & 53 & 640 \\
\hline GO:0060089 & molecular transducer activity & molecular function & 0.087474 & 53 & 640 \\
\hline GO:0006955 & immune response & biological process & 0.000127 & 19 & 108 \\
\hline GO:0002376 & immune system process & biological process & 0.000184 & 19 & 111 \\
\hline GO:0009259 & ribonucleotide metabolic process & biological process & 0.009166 & 16 & 122 \\
\hline GO:0009150 & purine ribonucleotide metabolic process & biological process & 0.017198 & 15 & 120 \\
\hline GO:0072521 & purine-containing compound metabolic process & biological process & 0.025628 & 15 & 126 \\
\hline GO:0009117 & nucleotide metabolic process & biological process & 0.026075 & 18 & 160 \\
\hline GO:0009119 & ribonucleoside metabolic process & biological process & 0.033152 & 14 & 119 \\
\hline GO:1901135 & carbohydrate derivative metabolic process & biological process & 0.035667 & 25 & 249 \\
\hline GO:0006753 & nucleoside phosphate metabolic process & biological process & 0.043746 & 18 & 170 \\
\hline GO:0007186 & G-protein coupled receptor signaling pathway & biological process & 0.047943 & 49 & 561 \\
\hline GO:0007606 & sensory perception of chemical stimulus & biological process & 0.049864 & 125 & 809 \\
\hline GO:0009116 & nucleoside metabolic process & biological process & 0.058703 & 14 & 129 \\
\hline GO:1901657 & glycosyl compound metabolic process & biological process & 0.058703 & 14 & 129 \\
\hline GO:0019637 & organophosphate metabolic process & biological process & 0.072145 & 21 & 218 \\
\hline GO:0055086 & nucleobase-containing small molecule metabolic process & biological process & 0.084247 & 18 & 185 \\
\hline GO:0007600 & sensory perception & biological process & 0.0031005 & 154 & 1023 \\
\hline GO:0071944 & cell periphery & cellular component & 0.024498 & 10 & 72 \\
\hline
\end{tabular}

findings of previous studies, which have shown that there are a large family of olfactory genes and that these are associated with CNVs in humans [45], mice, and dogs [46]. This apparent conservation of CNVs across mammalian species may be attributable, in part at least, to the fact that selective pressure might drive the acquisition or retention of specific gene dosage alterations, and that the gene families involved in sensory perception are typically rapidly evolving because they play key roles in the response of organisms to rapid changes in the environment and have been repeatedly detected in CNVRs of cattle, mouse, and dog genomes [47, 48]. The functional category enrichment of $\mathrm{CNV}$-harbored genes may be a reflection of their physiological role in the regulation of hypoxic adaption, species evolution and biodiversity.

The DCC gene encodes a netrin receptor, a key regulator in DCC/APPL-1/AKT pathway, which attenuates hypoxia-induced neuronal apoptosis and improves neurological function $[49,50]$. GSTCD, a member of a subgroup of the Glutathione S-Transferase (GST) gene family, plays a specific role in protecting against the products of oxidation stress, and its expression is induced by compounds associated with chemical stress and carcinogenesis [51]. MRPS28 is generally expressed in oxygen sensitive organs, including the brain, cerebellum, and kidney. Mitochondria are the primary energygenerating system in multiple eukaryotic cells, and are the energy supply centers for cellular processes, such as intermediary metabolism, calcium signaling, and apoptosis. MOGAT2, a member of MOGAT gene family, plays an important role in catalyzing the metabolism of triglycerides and is highly conserved in organisms. In this study, MOGAT2 was found to have different degrees of copy number amplification and deletion in the 16 yak populations. Heat map analysis also revealed that there is a significant difference in the $\mathrm{CNV}$ of the MOGAT2 among these individuals, which indicates that the mechanisms of fat metabolism and carbohydrate utilization are important to yak production and reproductive performance in the severe environment of the Qinghai-Tibetan Plateau. Furthermore, we found DEXI [52], CIITA [53], and SMYD1 [54] are key genes influencing the immune system and may reflect the substantially different diseases triggered by the parasites and arbovirus found at high altitudes. These results suggest that CNV is a key type of genetic variation that may play an important role 


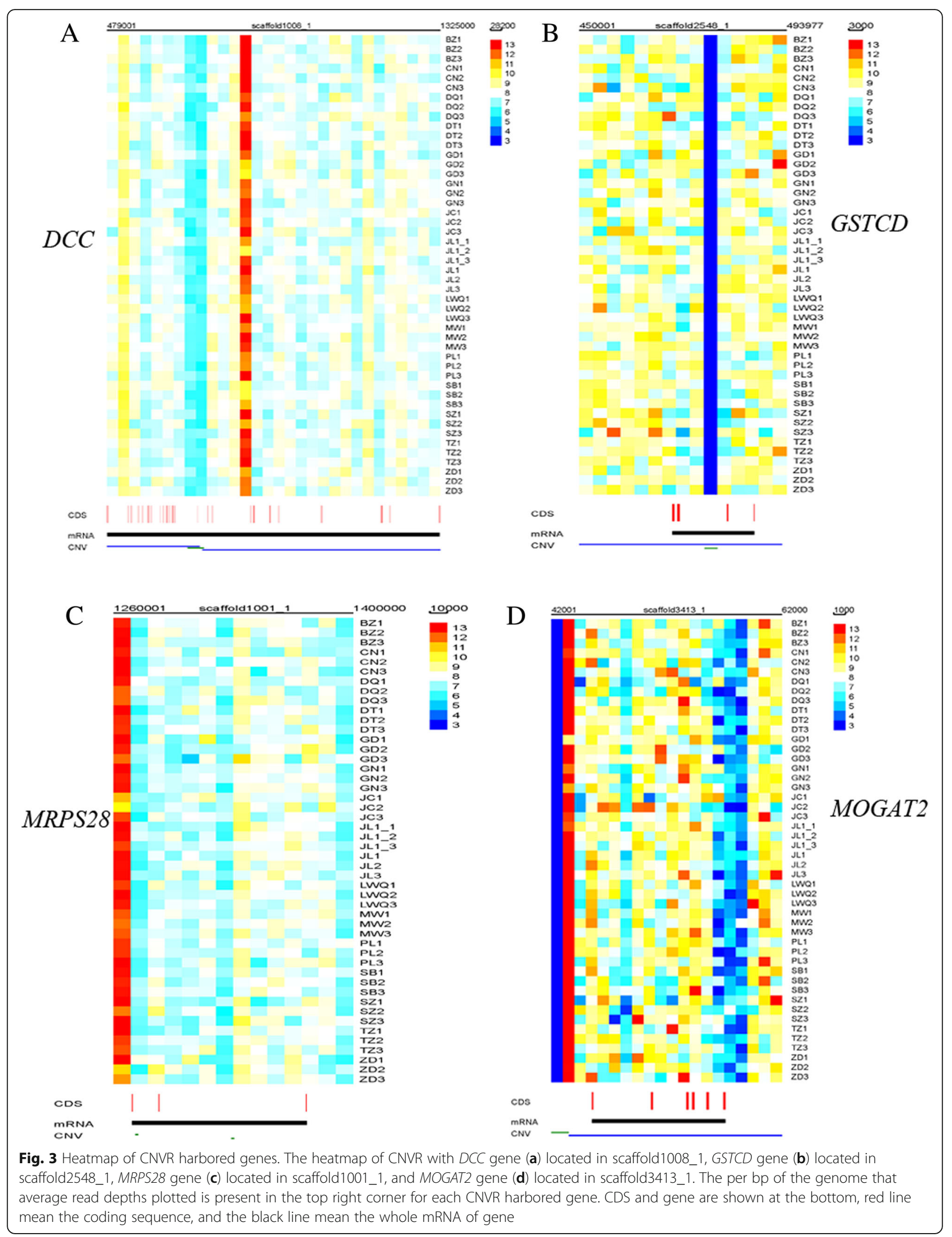




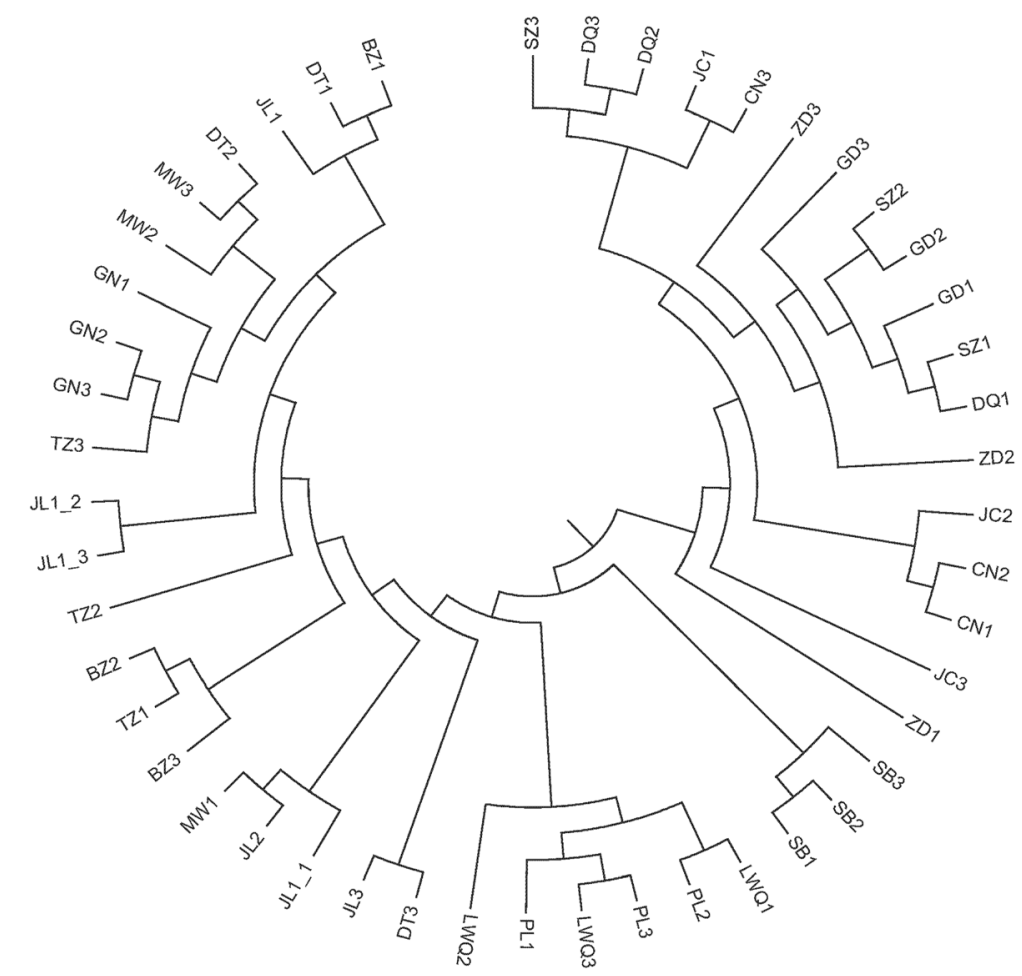

Fig. 4 The clustering map in 48 individuals based on CNVs

in yak adaptation to high-altitude environments, and thus it is desirable to carry out further research on the specific relationships or interaction mechanisms between the function of validated genes and CNV.

Our CNV clustering results based on individuals is consistent with the theory that the yaks have two different ancestral origins [55], although we also found that

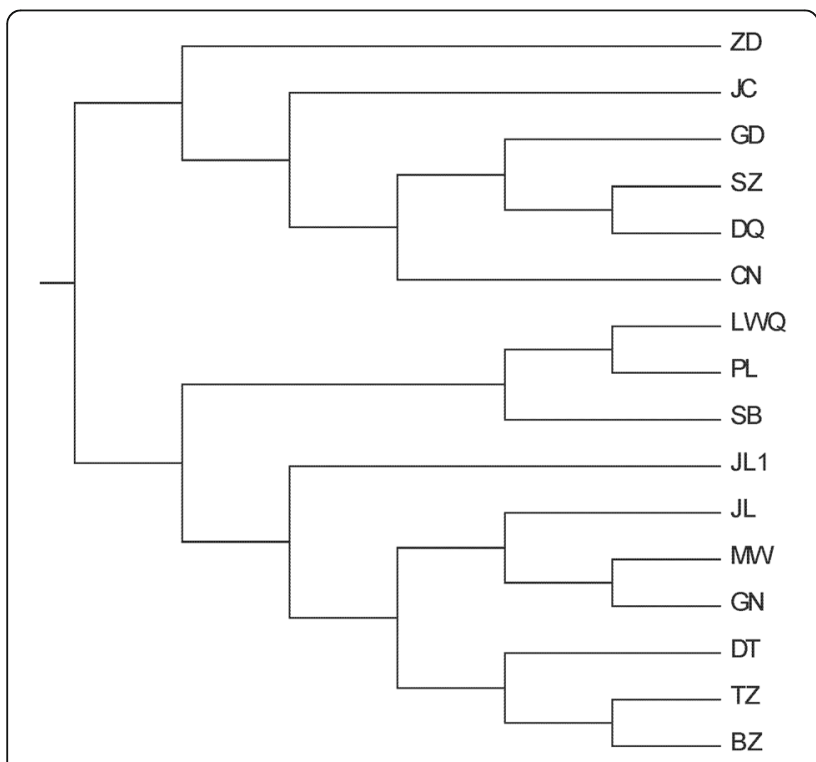

Fig. 5 The clustering map in 16 populations based on CNVs individuals within a single population showed cross clustering, only SB yaks were found to cluster together and at a distance from other yak populations, thereby indicating that CNVs may play an important role in the adaptation to hypoxia in SB yaks and their unique characters. In contrast to SNP-based cluster analysis, different yaks in the same population were found to cluster further from each other in clustering based on CNVs. For instance, two individuals from the LWQ yak population were clustered with the PL yak population, whereas the other individual in LWQ formed a solitary group. These findings are consistent with previous studies that have indicated that the characteristics of CNV-based clustering can show large variations between individuals, and that introgression plays a potentially important role in yak adaptation [56]. Indeed, cluster analysis of CNV variation has geographic distribution characteristics. For instance, TZ, DT, GN and MW and JL yaks are geographically close, which consequently enhances the opportunities for exchange genetic information among these populations, and is reflected in their closer clustering based on the coefficient of CNV. Similar results were observed for LWQ, SB and PL1 yaks. On the basis of these differences of $\mathrm{CNV}$ frequency among yaks, we hypothesize that yak CNVs are likely to arise independently in individuals/populations and contribute to individual/population differences and therefore are related to the population formation and adaptation, and gene 
communication exist between some populations. Different depths of sequence would markedly affect the detection rate and feature evaluation of CNV. To eliminate this imbalance as much as possible, cluster analyses were further performed based on populations, and the results indicated that the yak populations involved in this study were consistently classified into two continental groups.

\section{Conclusions}

In this study, we employed whole-genome sequencing to investigate the genome-wide CNV for 48 yaks in 16 populations. A total of 24,729 gain and 26,732 loss events, and 3174 CNVRs covering $163.8 \mathrm{M}(6.2 \%)$ of the yak genome were identified. These CNVs provide the largest source of these variations identified to date along with the highest-resolution CNVR and SD distribution maps for the 30 chromosomes of yak. The potential CNVRs contain 1374 functionally annotated genes and GO enrichment analysis revealed that these CNVR-harbored genes are largely related to oxidative phosphorylation, immune response, olfactory receptor activity, and sensory perception. Some novel CNVR-harbored genes, including $D C C$, GSTCD, MRPS28 and MOGAT2, are probably associated with the adaptation to high-altitude environments. In addition, the findings of our study support the hypothesis that the yak populations are mainly composed of two distinct ancestries. Taken together, our results constitute a valuable genome-wide variation resource of different yak populations for future work on phenotypic variation and breeding in yaks and provide insights into the mechanisms underlying yak genome evolution.

\section{Additional files}

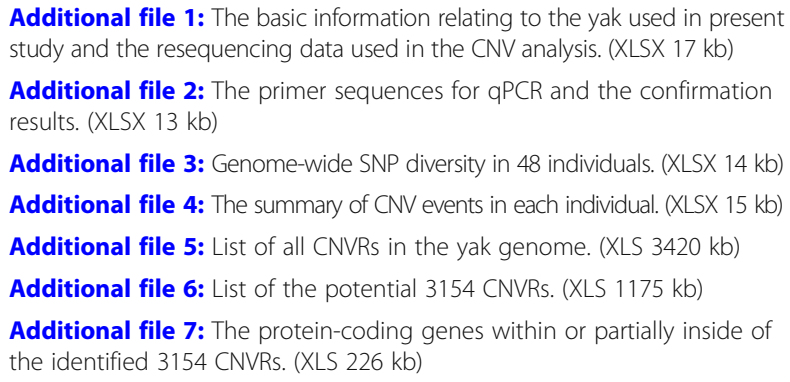

\section{Abbreviations}

BTF3: Basic transcription factor 3; BZ: Bazhou yak; CGH: Comparative genomic hybridization; CN: Cuona yak; CNVR: Copy number variation region; CNVs: Copy number variations; DCC: Deleted in colorectal cancer; DQ: Dingqing yak; DT: Datong yak; FISH: Fluorescent in situ hybridization; GD: Gongbujiangda yak; GN: Gannan yak; GO: gene ontology; GSTCD: glutathione S-transferase C-terminal domain containing; JC: Jinchuan yak; JL: Jiulong yak; JL1: Jiali yak; LWQ: Leiwuqi yak; MOGAT2: monoacylglycerol O-acyltransferase 2; MRPS28: mitochondrial ribosomal protein s28; MW: Maiwa yak; PL: Pali yak; qPCR: quantitative PCR; SB: Sibu yak; SDs: segmental duplications; SZ: Shenzha yak; TZ: Tianzhu yak; ZD: Zhongdian yak

\section{Acknowledgements}

The authors gratefully thank Dr. Deming Gou at Shenzhen University (Shenzhen, China) and Yu Jiang at Northwest A\&F University (Yangling, China) for taking technical support.

\section{Funding}

This study was supported by the Program of National Beef Cattle and Yak Industrial Technology System (CARS-37) in designing the study and sample collection, the Fundamental Research Funds for the Central Universities, Southwest Minzu University (2018NQN40) in analysis and interpretation of the data, and the Foundation of the Key Laboratory of Qinghai-Tibetan Plateau Animal Genetic Resource Reservation and Utilization (Southwest Minzu University), Ministry of Education, in writing the manuscript.

\section{Availability of data and materials}

The datasets supporting the conclusions of this article are included as Additional files 1, 2, 3, 4, 5, 6, and 7 .

\section{Authors' contributions}

HW, ZXC and JCZ conceived and designed the experiments, HW, DH and QMJ analyzed the data, HW, JWX and CFZ performed the experiments. HW wrote the paper. All authors read and approved the final manuscript.

\section{Ethics approval and consent to participate}

All animal experiments were conducted according to the regulations for the Administration of Affairs Concerning Experimental Animals (Ministry of Science and Technology, China, revised in June 2004) and approved by the Institution Animal Care and Use Committee in the Southwest Minzu University, Chengdu, China. We obtained written permission from the owner of the yak to take samples.

\section{Consent for publication}

Not applicable.

\section{Competing interests}

The authors declared that they have no competing interests.

\section{Publisher's Note}

Springer Nature remains neutral with regard to jurisdictional claims in published maps and institutional affiliations.

\section{Author details}

${ }^{1}$ Key Laboratory of Qinghai-Tibetan Plateau Animal Genetic Resource Reservation and Utilization (Southwest Minzu University), Ministry of Education, Chengdu 610000, People's Republic of China. ${ }^{2}$ State Key Laboratory of Barley and Yak Germplasm Resources and Genetic Improvement, Tibet Academy of Agricultural and Animal Husbandry Sciences, Lhasa 850000, People's Republic of China.

Received: 27 July 2018 Accepted: 11 January 2019

Published online: 18 January 2019

\section{References}

1. Abecasis GR, Altshuler D, Auton A, Brooks LD, Durbin RM, Gibbs RA, Hurles ME. McVean GA. A map of human genome variation from population-scale sequencing. Nature. 2010;467(7319):1061-73.

2. Ghosh S, Qu Z, Das PJ, Fang E, Juras R, Cothran EG, McDonell S, Kenney DG, Lear TL, Adelson DL, et al. Copy number variation in the horse genome. PLoS Genet. 2014;10(10):e1004712.

3. Kamath BM, Thiel BD, Gai X, Conlin LK, Munoz PS, Glessner J, Clark D, Warthen DM, Shaikh TH, Mihci E, et al. SNP array mapping of chromosome 20p deletions: genotypes, phenotypes, and copy number variation. Hum Mutat. 2009;30(3):371-8.

4. lacocca MA, Hegele RA. Role of dna copy number variation in dyslipidemias. Curr Opi Lipidol. 2018;29(2):125-32.

5. Hull RM, Cruz C, Jack CV, Houseley J. Environmental change drives accelerated adaptation through stimulated copy number variation. PLoS Biol. 2017;15(6):e2001333

6. Ruderfer DM, Hamamsy T, Lek M, Karczewski KJ, Kavanagh D, Samocha KE, Exome AC, Daly MJ, MacArthur DG, Fromer M, et al. Patterns of genic 
intolerance of rare copy number variation in 59,898 human exomes. Nat Genet. 2016:48(10):1107-11.

7. Prunier J, Caron S, Lamothe M, Blais S, Bousquet J, Isabel N, MacKay J. Gene copy number variations in adaptive evolution: the genomic distribution of gene copy number variations revealed by genetic mapping and their adaptive role in an undomesticated species, white spruce (Picea glauca) Mol Ecol. 2017;26(21):5989-6001.

8. Yang L, Xu L, Zhou Y, Liu M, Wang L, Kijas JW, Zhang H, Li L, Liu GE. Diversity of copy number variation in a worldwide population of sheep. Genomics. 2018;110(3):143-8.

9. Bickhart DM, Xu L, Hutchison JL, Cole JB, Null DJ, Schroeder SG, Song J, Garcia JF, Sonstegard TS, Van Tassell CP, et al. Diversity and populationgenetic properties of copy number variations and multicopy genes in cattle. DNA Res. 2016;23(3):253-62.

10. Kishimoto R, Tamada K, Liu X, Okubo H, Ise S, Ohta H, Ruf S, Nakatani J, Kohno N, Spitz F, et al. Model mice for 15q11-13 duplication syndrome exhibit late onset obesity and altered lipid metabolism. Hum Mol Genet. 2015;24(16):4559-72.

11. Chung RH, Chiu YF, Hung YJ, Lee WJ, Wu KD, Chen HL, Lin MW, Chen Yl, Quertermous T, Hsiung CA. Genome-wide copy number variation analysis identified deletions in SFMBT1 associated with fasting plasma glucose in a Han Chinese population. BMC Genomics. 2017;18(1):591.

12. Shlien A, Malkin D. Copy number variations and cancer. Genome Med. 2009; $1(6): 62$.

13. Wang P, Guan D, Yue P, Xu S, Gong L, Yuan Y. Copy number variations and its clinical significance of N-MYC gene in children with neuroblastic tumors. Journal of Clinical \& Pathological Research. 2017;11:2339-44.

14. Shwan NAA, Louzada S, Yang F, Armour JAL. Recurrent rearrangements of human amylase genes create multiple independent CNV series. Hum Mutat. 2017:38(5):532-9.

15. Zarrei M, Macdonald JR, Merico D, Scherer SW. A copy number variation map of the human genome. Nat Rev Genet. 2015;16(3):172-83.

16. Jiang J, Wang J, Wang H, Zhang Y, Kang H, Feng X, Wang J, Yin Z, Bao W, Zhang Q, Liu JF. Global copy number analyses by next generation sequencing provide insight into pig genome variation. BMC Genomics. 2014;15(1):593.

17. Fontanesi L, Martelli PL, Beretti F, Riggio V, Dall'Olio S, Colombo M, Casadio R, Russo V, Portolano B. An initial comparative map of copy number variations in the goat (Capra hircus) genome. BMC Genomics. 2010;11(1):639.

18. Jenkins GM, Goddard ME, Black MA, Brauning R, Auvray B, Dodds KG, Kijas JW, Cockett N, McEwan JC. Copy number variants in the sheep genome detected using multiple approaches. BMC Genomics. 2016;17(1):441.

19. Gao Y, Jiang J, Yang S, Hou Y2, Liu GE, Zhang S, Zhang Q, Sun D. CNV discovery for milk composition traits in dairy cattle using whole genome resequencing. BMC Genomics. 2017;18(1):265.

20. Sohrabi SS, Mohammadabadi M, Wu DD, Esmailizadeh A. Detection of breed specific copy number variations in domestic chicken genome. Genome. 2018;61(1):7-14.

21. Xu L, Cole JB, Bickhart DM, Song J, VanRaden PM, Sonstegard TS, Van Tassell CP, Liu GE. Genome wide CNV analysis reveals additional variants associated with milk production traits in Holsteins. BMC Genomics. 2014;15(1):683.

22. Yang Z, Connor EE, Wiggans GR, Lu Y, Tempelman RJ, Schroeder SG, Chen H, Liu GE. Genome-wide copy number variant analysis reveals variants associated with 10 diverse production traits in Holstein cattle. BMC Genomics. 2018;19(1):314.

23. Revilla M, Puig-Oliveras A, Castelló A, Crespo-Piazuelo D, Paludo E Fernández Al, Ballester M, Folch JM. A global analysis of CNVs in swine using whole genome sequence data and association analysis with fatty acid composition and growth traits. Plos One. 2017;12(5):e0177014.

24. Han JL, Yang M, Yue YJ, Guo T, Liu JB, Niu CE, Yang BH. Analysis of agouti signaling protein (ASIP) gene polymorphisms and association with coat color in Tibetan sheep (Ovis aries). Genet Mol Res. 2015;14(1):1200-9.

25. Lemos MVAD, Berton MP, Peripolli E, Gregório M, Peripollia E, Rafael M, Bianca F, Aline S, Angélica S, Lucia G, et al. Copy number variation regions in Nellore cattle: evidences of environment adaptation. Livest Sci. 2018;207:51-8.

26. Bickhart DM, Hou Y, Schroeder SG, Alkan C, Cardone MF, Matukumalli LK, Song J, Schnabel RD, Ventura M, Taylor JF, et al. Copy number variation of individual cattle genomes using next-generation sequencing. Genome Res. 2012;22(4):778-90.
27. Gao Z, Luo G, Ni B. Progress in mass spectrometry-based proteomics in hypoxia-related diseases and high-altitude medicine. OMICS. 2017;21(6):305-13.

28. Wang ZF, Shen X, Liu B, Su J, Yonezawa T, Yu Y, Guo S, Simon Y, Carles V, Hasegawa $\mathrm{M}$, et al. Phylogeographical analyses of domestic and wild yaks based on mitochondrial DNA: new data and reappraisal. J Biogeogr. 2010; 37(12):2332-44.

29. Zhang L, Jia S, Yang M, Xu Y, Li C, Sun J, Huang Y, Lan X, Lei C, Zhou Y, et al. Detection of copy number variations and their effects in Chinese bulls. BMC Genomics. 2014;15(1):480.

30. Grooms DL, Keilen ED. Screening of neonatal calves for persistent infection with bovine viral diarrhea virus by immunohistochemistry on skin biopsy samples. Clin Diagn Lab Immunol. 2002;9(4):898-900.

31. Ausubel FM, Brent R, Kingston RE, Mooree D, Seidman JG, Smith JA, Strunl K. Current protocols in molecular biology. New York, NY: John Wiley; 1993. p. 3-8.

32. Sambrook J, Russell D. Molecular cloning: a laboratory manual (3-volume set). Immunol. 2001:49:895-909.

33. Zhang X, Wang K, Wang L, Yang Y, Ni Z, Xie X, Shao X, Han J, Wan D, Qiu Q Genome-wide patterns of copy number variation in the Chinese yak genome. BMC Genomics. 2016;17(1):379.

34. Abyzov A, Urban AE, Snyder M, Gerstein M. CNVnator: an approach to discover, genotype and characterize typical and atypical cnvs from family and population genome sequencing. Genome Res. 2011;21(6):974-84.

35. Vandesompele J, De Preter K, Pattyn F, Poppe B, Van Roy N, De Paepe A, Speleman F. Accurate normalization of real-time quantitative RT-PCR data by geometric averaging of multiple internal control genes. Genome Biol. 2002;3(7):RESEARCH0034.

36. Liu GE, Hou YL, Zhu B, Jiang L, Cellamare A, Mitra A, Alexander LJ, Coutinho LL, Dell'Aquila ME, Gasbarre LC, et al. Analysis of copy number variations among diverse cattle breeds. Genome Res. 2010;20(5):693-703.

37. Ye J, Zhang Y, Cui H, Liu J, Wu Y, Cheng Y, Xu H, Huang X, Li S, Zhou A, et al. WEGO 2.0: a web tool for analyzing and plotting GO annotations, 2018 update. Nucleic Acids Res. 2018;46(W1):W71-5.

38. Glessner JT, Li J, Wang D, March M, Lima L, Desai A, Hadley D, Kao C, Gur $\mathrm{RE}$, Cohen $\mathrm{N}$, et al. Copy number variation meta-analysis reveals a novel duplication at 9p24 associated with multiple neurodevelopmental disorders. Genome Med. 2017;9(1):106.

39. Steinberg KM, Schneider VA, Graveslindsay TA, Fulton RS, Agarwala R, Huddleston J, Shiryev SA, Morgulis A, Surti U, Warren WC, et al. Single haplotype assembly of the human genome from a hydatidiform mole. Genome Res. 2014;24(12):2066-76.

40. Hou Y, Liu GE, Bickhart DM, Cardone MF, Wang K, Kim ES, Matukumalli LK, Ventura M, Song J, VanRaden PM, et al. Genomic characteristics of cattle copy number variations. BMC Genomics. 2011;12(1):127.

41. Ferrier DE, Martinez P. Evolutionary developmental genomics: at the 2008 meeting of the European society for evolutionary developmental biology. Genomics. 2010;95(5):247-9.

42. Halder A, Jain M, Chaudhary I. Suspected microdeletion syndromes and molecular cytogenetic techniques: an experience with 330 cases. Mol Cytogenet. 2014;7(S1):1-2.

43. Goidts V, Cooper DN, Armengol L, Schempp W, Conroy J, Estivill X, Nowak $\mathrm{N}$, Hameister $\mathrm{H}$, Kehrer-Sawatzki H. Complex patterns of copy number variation at sites of segmental duplications: an important category of structural variation in the human genome. Hum Genet. 2006;120(2):270-84.

44. Cantsilieris S, Baird PN, White SJ. Molecular methods for genotyping complex copy number polymorphisms. Genomics. 2013:101(2):86-93.

45. Young JM, Endicott RLM, Parghi SS, Walker M, Kidd JM, Trask BJ. Extensive copy-number variation of the human olfactory receptor gene family. Am J Hum Genet. 2008;83(2):228-42.

46. Hughes GM, Boston EM, Finarelli JA, Murphy WJ, Higgins DG, Teeling EC. The birth and death of olfactory receptor gene families in mammalian niche adaptation. Mol Biol Evol. 2018;35(6):1390-406.

47. Pezer Ž, Harr B, Teschke M, Babiker H, Tautz D. Divergence patterns of genic copy number variation in natural populations of the house mouse (Mus musculus domesticus) reveal three conserved genes with major populationspecific expansions. Genome Res. 2015;25(8):1114-24.

48. Paudel Y, Madsen O, Megens HJ, Frantz LA, Bosse M, Bastiaansen JW, Crooijmans RP, Groenen MA. Evolutionary dynamics of copy number variation in pig genomes in the context of adaptation and domestication. BMC Genomics. 2013;14(1):449.

49. Xie Z, Huang L, Enkhjargal B, Reis C, Wan W, Tang J, Cheng Y, Zhang JH. Intranasal administration of recombinant Netrin-1 attenuates neuronal 
apoptosis by activating DCC/APPL-1/AKT signaling pathway after subarachnoid hemorrhage in rats. Neuropharmacology. 2017;119:123-33.

50. Son TW, Yun SP, Yong MS, Seo BN, Ryu JM, Youn HY, Oh YM, Han HJ. Netrin-1 protects hypoxia-induced mitochondrial apoptosis through HSP27 expression via DCC- and integrin a6ß4-dependent Akt, GSK-3 $\beta$, and HSF-1 in mesenchymal stem cells. Cell Death Dis. 2013;4(3):e563.

51. Yang Y, Sharma R, Sharma A, Awasthi S, Awasthi YC. Lipid peroxidation and cell cycle signaling: 4-hydroxynonenal, a key molecule in stress mediated signaling. Acta Biochim Pol. 2003;50(2):319-36.

52. Edgar AJ, Birks EJ, Yacoub MH, Polak JM. Cloning of dexamethasoneinduced transcript: a novel glucocorticoid-induced gene that is upregulated in emphysema. Am J Respir Cell Mol Biol. 2001;25(1):119-24.

53. Mehta NT, Truax AD, Boyd NH, Greer SF. Early epigenetic events regulate the adaptive immune response gene CIITA. Epigenetics. 2011;6(4):516-25.

54. Maysaa D, Nicholas S, Li C, Yang Z. SMYD proteins in immunity: dawning of a new era. Aims Biophysics. 2016;3(4):450-5.

55. Lai SJ, Chen SY, Liu YP, Yao YG. Mitochondrial DNA sequence diversity and origin of Chinese domestic yak. Anim Genet. 2007;38(1):77-80.

56. Wu DD, Ding XD, Wang S, Wójcik JM, Zhang Y, Tokarska M, Li Y, Wang MS, Faruque $\mathrm{O}$, Nielsen $\mathrm{R}$, et al. Pervasive introgression facilitated domestication and adaptation in the Bos species complex. Nat Ecol Evol. 2018;2(7):1139-45.

Ready to submit your research? Choose BMC and benefit from:

- fast, convenient online submission

- thorough peer review by experienced researchers in your field

- rapid publication on acceptance

- support for research data, including large and complex data types

- gold Open Access which fosters wider collaboration and increased citations

- maximum visibility for your research: over $100 \mathrm{M}$ website views per year

At $\mathrm{BMC}$, research is always in progress.

Learn more biomedcentral.com/submissions 